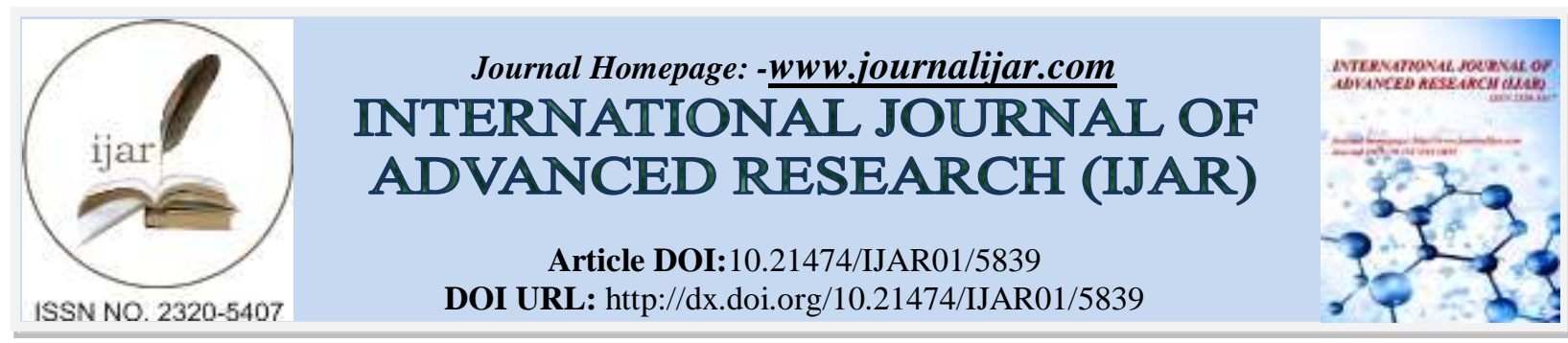

RESEARCH ARTICLE

\title{
SECOND PRIMARY TUMOR OF THE TONGUE - A CASE REPORT.
}

Dr. Asha R Iyengar ${ }^{1}$, Dr. SeemaPatil ${ }^{2}$, Dr.Rahamath Fatima ${ }^{3}$, Dr. Vani M.H ${ }^{4}$ and Dr. Subhash B.V ${ }^{5}$.

1. Professor and HOD, Department of Oral Medicine and Radiology, D.A.P.M.R.V Dental College.

2. Reader, Department of Oral Medicine and Radiology, D.A.P.M.R.V Dental College.

3. Post graduate student, Department of Oral medicine and Radiology, D.A.P.M.R.V Dental College.

4. Post graduate student, Department of Oral Medicine and Radiology, D.A.P.M.R.V Dental College, Bangalore.

5. Reader, Department of Oral medicine and Radiology, D.A.P.M.R.V Dental College.

\section{Manuscript Info}

(.........................

Manuscript History

Received: 11 September 2017

Final Accepted: 13 October 2017

Published: November 2017

\section{Key words:-}

Nasopharyngeal carcinoma, squamous cell carcinoma, second primary cancer, radiotherapy, field cancerization.

\section{Abstract}

Nasopharyngeal carcinoma (NPC) is a tumor arising from the epithelial cells lining the nasopharynx. The development of a second primary tumor in subjects with a previous history of nasopharyngeal carcinoma is a rare event. The most common second primary cancer which can occur is sarcoma $(0.14 \%)$, followed by leukemia and squamous cell carcinoma. In the present case, a second primary tumor (squamous cell carcinoma) on right lateral border of tongue in a 64 year old male patient treated for nasopharyngeal carcinoma with radiotherapy and chemotherapy 5 years previously is discussed.

Copy Right, IJAR, 2017,. All rights reserved.

\section{Introduction:-}

Nasopharyngeal carcinoma (NPC) is a tumor arising from the epithelial cells lining the nasopharynx. ${ }^{1}$ The development of a second primary malignant tumor in subjects with a previous history of nasopharyngeal carcinoma is unusual. ${ }^{2}$ The most common second primary cancer which can occur is sarcoma $(0.14 \%)$, followed by leukemia and squamous cell carcinoma. ${ }^{2}$ These second primaries can occur in the lung, oesophagus and also in the head and neck region. ${ }^{2}$ In the oro-pharyngeal region, tongue is a common site of involvement $(1.8 \%){ }^{2}$ The proposed pathogenic mechanisms of the development of second primary cancers include radiotherapy, field cancerization and common risk factors. ${ }^{2}$ The present case is of second primary tumor (squamous cell carcinoma) on right lateral border of tongue in a 64 year old male patient treated for nasopharyngeal carcinoma.

\section{Case Report:-}

A 64 year old male visited the department of Oral medicine and Radiology with a complaint of frequent biting of the right side of the tongue while eating and talking since 6 months. He had visited several dental practitioners for the management of the same and coronoplasty of maxillary and mandibular posterior teeth was performed. However, there was no relief. He had visited an oncology centre 2 days prior to visiting the present institution for a PET-CT scan. The scan was advised as a part of the follow-up protocol for treated nasopharyngeal carcinoma that was diagnosed 5 years ago. Patient had undergone radiotherapy and chemotherapy for the same. The treatment was completed 4 years ago. An increased FDG uptake was noted on PET-CT with regard to the right lateral aspect of tongue.

He was advised partial glossectomy by the oncosurgeon for the same. 
Patient was diabetic since 15 years and the condition was under control. History of weight loss ( $7 \mathrm{kgs})$ was noted in the past 6 months.

Patient gave history of chewing pan masala 2 to 3 packets in a day for about 15 years. He would chew for 15 to 20 min and spit it out. Patient had stopped the habit ever since nasopharyngeal carcinoma was diagnosed. On general examination patient was cooperative, moderately built, with normal gait and posture. No signs of pallor, cyanosis, icterus, edema and all the vital signs were within normal range.

On intra oral examination indentation of teeth was noted on right lateral aspect of tongue irt 46 . Beneath the indentation, a solitary, deep ulcer, irregular in shape measuring $5 \times 6 \mathrm{~mm}$ was noted. The floor was covered by yellowish white slough, the margins were well defined. Surrounding area appeared erythematous. There was no spontaneous bleeding or discharge. There was no restriction of tongue movements. The ulcer was tender on palpation. There was no induration of base and surrounding area. No bleeding or discharge was noted on palpation. (figure 1)

Based on history and clinical examination, a provisional diagnosis of non healing traumatic ulcer wrt right lateral border of the tongue was given.

Differential diagnosis of squamous cell carcinoma (second primary tumour), traumatic ulcerative granuloma and necrotizing sialometaplasia was considered.

PET-CT scan did not reveal any recurrent nasopharyngeal mass and significant cervical lymphadenopathy. A $1.7 \mathrm{X}$ $0.8 \mathrm{~cm}$ poorly marginated focus of metabolic activity was noted in the right lateral margin of the posterior tongue noted suggestive of neoplastic etiology. Regression in size and metabolic activity of mediastinal and hilar lymph nodes as compared to previous year PET-CT report was noted. (figure 2)

Biopsy was performed and histopathological report revealed the hematoxylin and eosin stained sections show keratinized stratified squamous epithelium with epithelial dysplasia. The malignant epithelial cells were seen invading into the connective tissue in the form of small islands and nests. There was presence of keratin pearls, few individual cell keratinization, nuclear and cellular pleomorphism and mitotic figures (both normal and abnormal). The surrounding stroma comprised of fibrous connective tissue with many blood vessels and chronic inflammatory cells. Few tumour giant cells were also evident. The features were suggestive of squamous cell carcinoma.( figure 3) A final diagnosis of second primary squamous cell carcinoma in respect to right lateral border of tongue was considered. Based on the PET-CT and histopathological report the patient was advised partial glossectomy.

Figure 1:- Intraoral photograph of the ulcer (arrow head) noted on right lateral aspect of tongue and indentation of teeth above it (arrow).

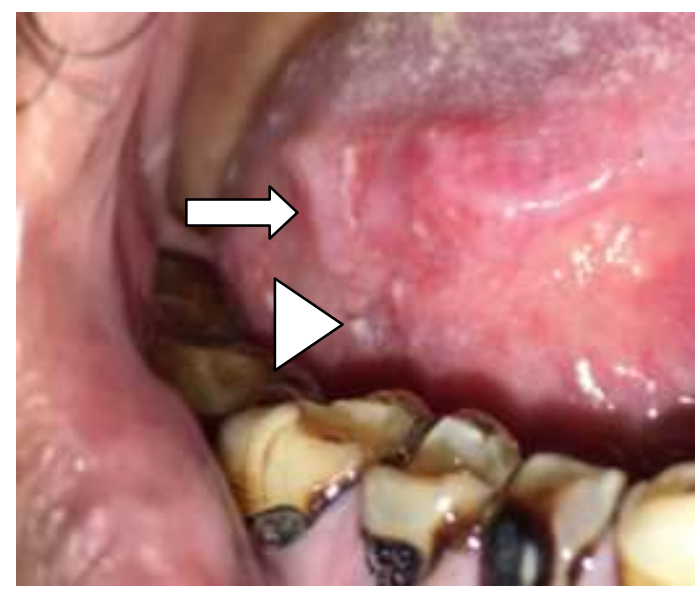


Figure 2:- PET-CT scan (axial section) showing increased metabolic activity on right lateral aspect of tongue.

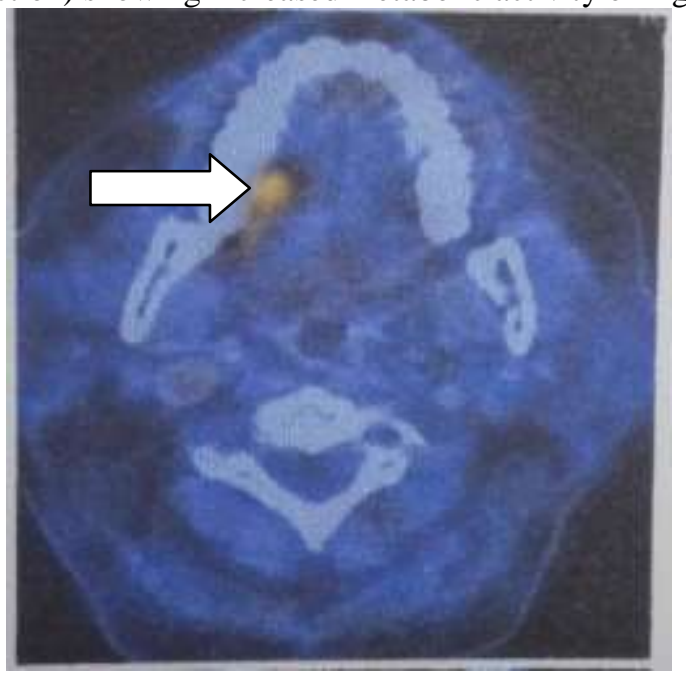

Figure 3:- Photomicrograph showing islands and nests of epithelial cells (arrow head) invading into connective tissue showing features of malignancy. Prominent keratin pearl formation (arrow) is seen (H\&E, 4X)

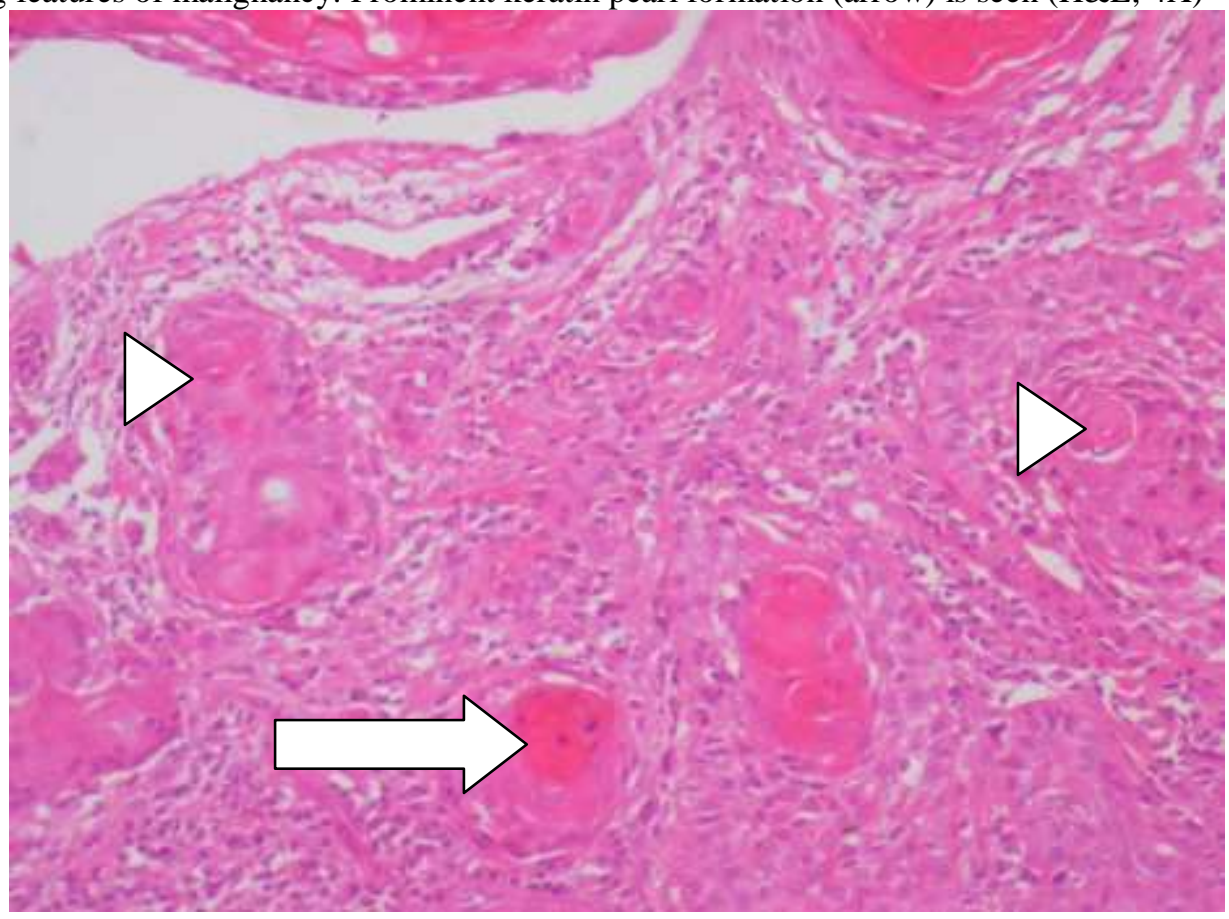

Table 1:- Comparison of squamous cell carcinoma of tongue in NPC survivor and non NPC. survivor.

\begin{tabular}{|l|l|}
\hline TSCC (NPC survivor ) & Sporadic TSCC (non NPC survivor) \\
\hline Age predilection for individuals less than $40 \mathrm{yrs}$ & $\begin{array}{l}\text { Age predilection for individuals greater than } 40 \\
\text { Female predilection }\end{array}$ \\
Site predilection for dorsum and lateral tongue & Site predilection for ventral tongue \\
\hline Tumor size smaller & Tumor size larger \\
Nodal involvement is less likely & Nodal involvement is common \\
\hline There is limited tumor budding & $\begin{array}{l}\text { There is extensive tumor budding and high risk pattern } \\
\text { Of invasion }\end{array}$ \\
\hline
\end{tabular}




\section{Discussion:-}

Nasopharyngeal carcinoma (NPC) is a tumor arising from the epithelial cells that line the nasopharynx. ${ }^{1}$ NPC is prevalent in China, Southeast Asia, Mediterranean basin and Alaska and an incidence of 2 per million has been reported in these regions. ${ }^{1}$

In India, the incidence is 0.9 per million, ${ }^{1}$ except in the Northeastern region $(1.82 \%) .{ }^{4}$ A number of etiologic factors such as race, genetics, environmental factors namely nitrosamines present in preserved /salted and fermented food, occupational exposure to wood dust, formaldehyde, chemical fumes and Epstein-Barr virus (EBV) have been implicated in the etiology of NPC. ${ }^{5}$

NPC occurs in both children and adults. It has definite gender predilection with a male to female ratio of 3:1. ${ }^{5}$ Currently, WHO classifies nasopharyngeal carcinoma (NPC) into keratinizing carcinoma (squamous cell carcinoma), seen in older individuals, non-keratinizing carcinoma (differentiated and undifferentiated variants) seen in children and adolescents and basaloid SCC. ${ }^{3}$ Symptoms vary according to the location of the tumor such as epistaxis, obstruction and discharge when the mass is in nasopharynx, decreased hearing and tinnitus when the tumour is located in the eustachian tube. Skull base involvement with impairment of the 5th and 6th cranial nerves causes headache, diplopia, facial pain, and paresthesia. ${ }^{3}$

Diagnosis of NPC is made by clinical evaluation of the size and location of tumor and cervical lymph nodes, neurological examination of cranial nerves, computed tomography (CT)/magnetic resonance imaging (MRI) scan of the head and neck to assess base of skull erosion, detection of EBV viral capsid antigen and EBV DNA and biopsy of either the lymph nodes or primary tumor for histological examination. NPC has characteristic features on PET/CT scans. It has an irregular or spherical shape with sharp edges. ${ }^{1}$

The tumour most frequently metastasizes to bone (70-80\%), followed by viscera (30\%) and at lower rates to extracervical lymph-nodes $(18 \%)$ such as axillary, mediastinal, pelvic, or inguinal. ${ }^{6}$

The mainstay of management for stages I and II is radiotherapy (RT), patients with stage III have been treated with concurrent chemotherapy and radiotherapy and stage $1 \mathrm{~V}$ with palliative chemotherapy. ${ }^{3}$

The development of a second primary malignant tumor (SMT) is an unusual but not a rare event. Malignancies that develop in these patients include soft tissue sarcoma,osteosarcoma, squamous cell carcinoma (SCC), leukaemia and neuroendocrine carcinomas. ${ }^{8}$ These tumours may develop in head and neck, oesophagus, lung and stomach. ${ }^{8}$ The most common histological type of second primary cancer is sarcoma $(0.14 \%)^{6}$ and the most common site of localization is oral-pharyngeal region. ${ }^{2}$ In the oropharyngeal region, the tongue is a common site of involvement $(1.8 \%) .{ }^{2}$ The proposed pathogenic mechanisms of second primary cancers include radiotherapy, field cancerization and impaired immune surveillance.

The incidence of radio-induced cancer after treatment of NPC varies from 0.04 to $7 \%$ in literature. ${ }^{2}$ Generally, posterior dorsum and tongue base are included in the field of external beam radiation for the tumor and cervical nodes. The occurrence of second primaries have been reported with the use of both medium and high dose of conventional therapy (2D) and 3D conformal and IMRT. ${ }^{8}$ Long term effects of radiotherapy in the form of indirect damage to blood vessels are thought to be responsible for occurrence of second primaries. ${ }^{8}$

Certain criteria have been proposed by researchers with regards to the eligibility for diagnosis of radio induced cancers which includes prior history of radiotherapy, secondary cancer within previously irradiated field, histologic confirmation of second malignancy and if the latency period between irradiation and second malignant tumor is atleast 1 year. $^{2}$

Field cancerization does not appear to be significant in the development of second primary tumors as NPCs and oral cancers do not share the same risk factors. NPCs are associated with genetic, environmental factors and EBV where as oral cancers are strongly associated with tobacco and alcohol. ${ }^{2}$

A reduced lymphocyte host response (LHR) has been found in patients with NPC suggesting it to be one of the contributing factors. ${ }^{2}$ 
In the present case, radiotherapy probably had a role in inducing SCC. Secondly, the patient had a habit of tobacco chewing for approximately 15 years which could be responsible for occurrence of SCC. Although, the patient had quit the habit 5 years ago, it has been observed in studies that the effect of tobacco can last upto10 years after discontinuation of habit.

The features of squamous cell carcinoma occurring on the tongue (TSCC) in a subject with a history of nasopharyngeal carcinoma differs when it occurs in a subject with no prior history of nasopharyngeal carcinoma. (table 1).

Most of the features of TSCC in the present case are in accordance with case reports reported in literature except with regards to age and gender. In previous reports, TSCC occurred mostly in females younger than 40 years but in the present case, it was found in a 64 year old male. Features in the present case such as location (lateral border of tongue), smaller size $(5 \times 6 \mathrm{~mm})$, absence of nodal involvement, tumour budding and lack of aggressiveness were in similarity with literature.

TSCC occurring in NPC survivor are usually managed by surgery. ${ }^{9}$ For non resectable tumors, palliative chemotherapy can be performed. ${ }^{9} \mathrm{Re}$ - irradiation has also been reported for few cases. ${ }^{9}$

\section{Conclusion:-}

It has been suggested that the tongue should be spared as much as possible during radiotherapy of NPCs in order to reduce the incidence of second primaries. Therefore, long term follow up of NPC cases treated with radiotherapy has been recommended. The occurrence of TSCC in the present case cannot be associated with radiotherapy alone as other contributing factors such as tobacco usage was also present. Hence, long term follow up is necessary for all cases of NPC irrespective of usage of radiotherapy as one of the treatment modalities.

\section{References:-}

1. Brennan B. Nasopharyngeal carcinoma (2006). Orphanet Journal of Rare Diseases; 1:23

2. Mnejja.W, Fourati N, Siala W, Farhat L, Elloumi F, Ghorbel A, Frikha M, Daoud J(2014). Tongue cancer after primary radiotherapy for nasopharyngeal carcinoma: Three cases report and literature review. J Nasopharyng Carcinoma 2014; 1(19): e19.

3. Petersson F (2015). Nasopharyngeal carcinoma: A review. SeminDiagnPathol; 32(1):54-73.

4. Kataki AC, Simons M, Das AK, Sharma K, Mehra NK (2011). Nasopharyngeal carcinoma in the northeastern states. Chin J Cancer; 30(2):106-13

5. Thompson L (2007). Update on Nasopharyngeal Carcinoma. Head and Neck Pathol; 1:81-6

6. Bensouda Y, Kaikani W, Ahbeddou N, Rahhali R, Jabri M, Mrabti H, Boussen H, Errihani H(2011). Treatment for metastatic nasopharyngeal carcinoma. Eur Ann Otorhinolaryngol Head Neck Dis;128(2):79-85

7. Mohan M, Jagannathan N (2014). Oral field cancerization: an update on current concepts. Oncol Rev; 8(1): 244.

8. Sun C, Hua Z, Zhonga Z, Jiang Y, Fei J, Xi F, Li X, Song M, Li W(2014). Clinical and prognostic analysis of second primary squamous cell carcinoma of the tongue after radiotherapy for nasopharyngeal carcinoma. $\mathrm{Br}$ J Oral MaxillofacSurg; 52(8):715-20.

9. Zhang P, Zhang L, Liu H, Zhao L, Li Shen J, Liu Q,Liu MZ, Xi M (2017). Clinicopathologic Characteristics and Prognosis of Tongue Squamous Cell Carcinoma in Patients with and without a History of Radiation for Nasopharyngeal Carcinoma: A Matched Case-Control Study. Cancer Res Treat; 49(3): 695-705. 